\title{
A Brief History of Regularisation
}

\author{
S. Mikkola \\ Tuorla Observatory, University of Turku, Väisäläntie 20, Piikkiö, Finland \\ email: seppo.mikkola@utu.fi
}

\begin{abstract}
The various methods for regularisation of the gravitational few-body problem, from the coordinate transformation by the Kustaanheimo-Stiefel method to the more recent methods of algorithmic regularisation, are reviewed. Numerical comparisons of the performance of the methods are presented and future research suggested.
\end{abstract}

Keywords. stellar dynamics, methods: n-body simulations, methods: numerical

\section{Introduction}

Close encounters of stars are common in dense stellar systems. Loss of precision was discovered by in the first attempts to simulate few-body systems (von Hoerner 1960, 1963) using the Newtonian form of equations of motion. This phenomenon is due to the $1 / r^{2}$ singularity in the gravitational force at distance $r$. In addition to the force value variation the roundoff error is a significant source of computational difficulties.

Numerical studies became feasible after the publication of the two-body regularization method by Kustaanheimo \& Stiefel (1965). Subsequently this KS-transformation became popular for treating dominant two-body interactions (e.g. Szebechely \& Peters 1967; Aarseth 1972). Techniques to apply KS in multiparticle systems were developed by many authors (Aarseth \& Zare 1974; Zare 1974; Heggie 1974; Mikkola 1985; Mikkola \& Aarseth 1993). A major reference for these methods is the book by Aarseth (2003).

More recently an entirely new way for regularization was discovered in 1999 (Mikkola \& Tanikawa 1999ab, Preto \& Tremaine 1999). This, algorithmic regularization, does not require coordinate transformation but a time transformation, the leapfrog algorithm and extrapolation to zero step size (Bulirsch \& Stoer 1966). Versions of few-body codes written using algorithmic regularization are more efficient than KS-based versions in case of very large mass ratios.

\section{Events and methods in the history of regularization}

- Poincare's time transformation: More than a century ago Poincare [cited in Siegel 1956, p.35] introduced a technique to transform the independent variable in a Hamiltonian system.

Let $H(\mathbf{p}, \mathbf{q}, t)$ be a Hamiltonian. One takes the time to be a canonical coordinate $\left(t=q_{0}\right)$ by adding the momentum of time $p_{0}(=-E)$ to the Hamiltonian (here $E=H=$ energy $=$ constant $)$. Defining $d t=g(\mathbf{p}, \mathbf{q}) d s$, where $g>0$, one gets a new Hamiltonian

$$
\Gamma=g(\mathbf{p}, \mathbf{q})\left[H\left(\mathbf{p}, \mathbf{q}, q_{0}\right)+p_{0}\right]
$$


which gives the equations of motion

$$
\begin{aligned}
& \frac{d t}{d s}=\frac{\partial \Gamma}{\partial p_{0}}=g \\
& \frac{d q}{d s}=\frac{\partial \Gamma}{\partial p} ; \quad \frac{d p}{d s}=-\frac{\partial \Gamma}{\partial q}
\end{aligned}
$$

In two-body regularisation one normally uses $g=r$ (where $r$ is the distance). For multiparticle systems initially the product of distances were proposed, but later it was found that the inverse of potential $U=\sum_{i<j} m_{i} m_{j} / r_{i j}$ or the inverse of the Lagrangian $L=T+U$ (where $T=$ the kinetic energy) are more appropriate, e.g.

$$
g=1 / U \quad \text { or } \quad g=1 / L
$$

- Levi-Civita (1920) published the regularisation in 2D.

$$
x=Q_{1}^{2}-Q_{2}^{2} ; y=2 Q_{1} Q_{2}, \text { or } x+i y=\left(Q_{1}+i Q_{2}\right)^{2}
$$

which gives for the two-body Hamiltonian (with $g=r=\mathbf{Q}^{2}$ )

$$
\Gamma=r\left(\frac{1}{2} \mathbf{p}^{2}-M / r-E_{0}\right)=\frac{1}{8} \mathbf{P}^{2}-E_{0} \mathbf{Q}^{2}-M,
$$

which is the Hamiltonian of a harmonic oscillator.

- Kustaanheimo \& Stiefel (1965) published their new regularization method which uses a transformation from $4 \mathrm{D}$ to $3 \mathrm{D}$.

In matrix formulation the KS-transformation of coordinates $\mathbf{r}$ and momenta $\mathbf{p}$ may be written

$$
\mathbf{r}=\widehat{\mathbf{Q}} \mathbf{Q} ; \quad \mathbf{p}=\widehat{\mathbf{Q}} \mathbf{P} /\left(2 Q^{2}\right)
$$

Here $\widehat{\mathbf{Q}}$ is the KS-matrix (e.g. Stiefel \& Scheifele 1972 p. 24)

$$
\widehat{\mathbf{Q}}=\left(\begin{array}{rrrr}
Q_{1} & -Q_{2} & -Q_{3} & Q_{4} \\
Q_{2} & Q_{1} & -Q_{4} & -Q_{3} \\
Q_{3} & Q_{4} & Q_{1} & Q_{2} \\
Q_{4} & -Q_{3} & Q_{2} & -Q_{1}
\end{array}\right)
$$

The time transformation

gives the new Hamiltonian

$$
\frac{d t}{d s}=R=\mathbf{Q}^{2}
$$

$$
\Gamma=R(H-E)=\frac{1}{8} \mathbf{P}^{2}-M-E \mathbf{Q}^{2},
$$

where $H=\frac{1}{2} p^{2}-M / r$ is the two-body Hamiltonian and $E=H(0)$ is the numerical value of the energy.

- Aarseth \& Zare (1974) published a three-particle regularization method. The two shortest distances are regularised with the Kustaanheimo-Stiefel method and the arrangement is appropriately updated when necessary.

Taking the vectors $\mathbf{R}_{1}$ and $\mathbf{R}_{2}$ (see the figure) as new canonical coordinates, the generating function takes the form

$$
S=\mathbf{W}_{1} \cdot \mathbf{R}_{1}+\mathbf{W}_{2} \cdot \mathbf{R}_{2}=\mathbf{W}_{1} \cdot\left(\mathbf{r}_{1}-\mathbf{r}_{3}\right)+\mathbf{W}_{2} \cdot\left(\mathbf{r}_{2}-\mathbf{r}_{3}\right)
$$




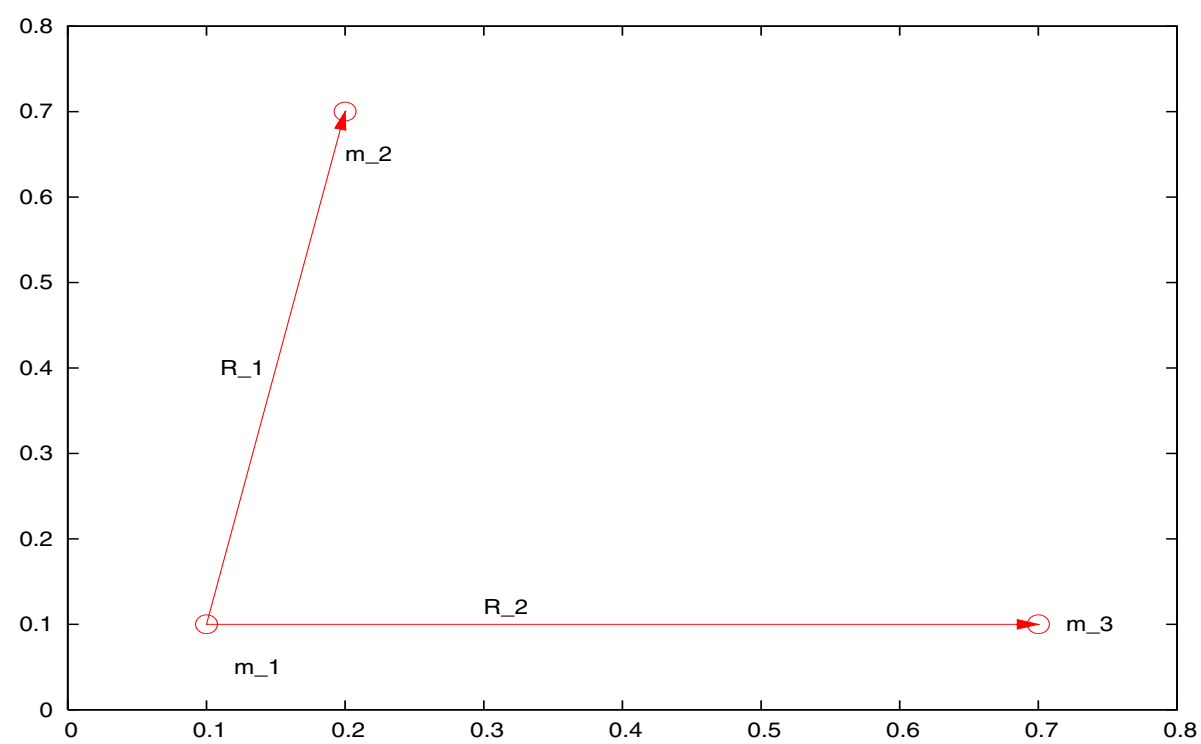

Figure 1. Regularised vectors in the Aarseth-Zare method.

and the physical momenta are $\mathbf{p}_{1}=\partial S / \partial \mathbf{r}_{1}=\mathbf{W}_{1}, \quad \mathbf{p}_{2}=\partial S / \partial \mathbf{r}_{2}=\mathbf{W}_{2}$ and $\mathbf{p}_{3}=$ $\partial S / \partial \mathbf{r}_{3}=-\mathbf{W}_{1}-\mathbf{W}_{2}$.

KS-transformation and the time transformation $d t=R_{1} R_{2} d s=Q_{1}^{2} Q_{2}^{2} d s$, gives the Hamiltonian

$$
\begin{aligned}
\Gamma= & \frac{1}{8 \mu_{13}} Q_{2}^{2} \mathbf{P}_{1}^{2}+\frac{1}{8 \mu_{23}} Q_{1}^{2} \mathbf{P}_{2}^{2}+\frac{1}{4 m_{3}} \mathbf{P}_{1}^{T} \widehat{\mathbf{Q}}_{1}^{T} \widehat{\mathbf{Q}}_{2} \mathbf{P}_{2} \\
& -m_{1} m_{3} Q_{2}^{2}-m_{2} m_{3} Q_{1}^{2}-m_{1} m_{2} Q_{1}^{2} Q^{2}{ }_{2} / R-E Q_{1}^{2} Q_{2}^{2},
\end{aligned}
$$

where $\mu_{k 3}=m_{k} m_{3} /\left(m_{k}+m_{3}\right)$, and $E$ is the total energy. The equations of motion $\mathrm{i}$

$$
\mathbf{P}_{k}^{\prime}=-\frac{\partial \Gamma}{\partial \mathbf{Q}_{k}} ; \quad \mathbf{Q}_{k}^{\prime}=\frac{\partial \Gamma}{\partial \mathbf{P}_{k}} ; \quad t^{\prime}=Q_{1}^{2} Q_{2}^{2}
$$

are regular with respect to collisions between the body $m_{3}$ and any one of the two bodies $m_{1}$ and $m_{2}$ A switching of the reference body is carried out whenever the singular distance $R$ becomes the smallest. In case of external perturbations the three-body energy $E$ is no longer a constant, but must be obtained by integration.

- Heggie (1974) Discovered a global N-body regularization using KS-transformations. In this method each interparticle vector is a (formally) independent variable. The Hamiltonian is $H=\sum_{i=1}^{N} \frac{1}{2 m_{i}} w_{i}^{2}-\sum_{i=1}^{N-1} \sum_{j=i+1}^{N} \frac{m_{i} m_{j}}{r_{i j}}$. Heggie (1974) uses $\mathbf{r}_{i j}=$ $\mathbf{r}_{j}-\mathbf{r}_{i}$ as new dependent variables. The generating function

$$
S=\sum_{i=1}^{N-1} \sum_{j=i+1}^{N} \mathbf{w}_{i j} \cdot\left(\mathbf{r}_{j}-\mathbf{r}_{i}\right)
$$

defines the new momenta $\mathbf{w}_{i j}$. A proof for the correctness of such an increase of degrees of freedom is also given in Heggie's (1974) article. 


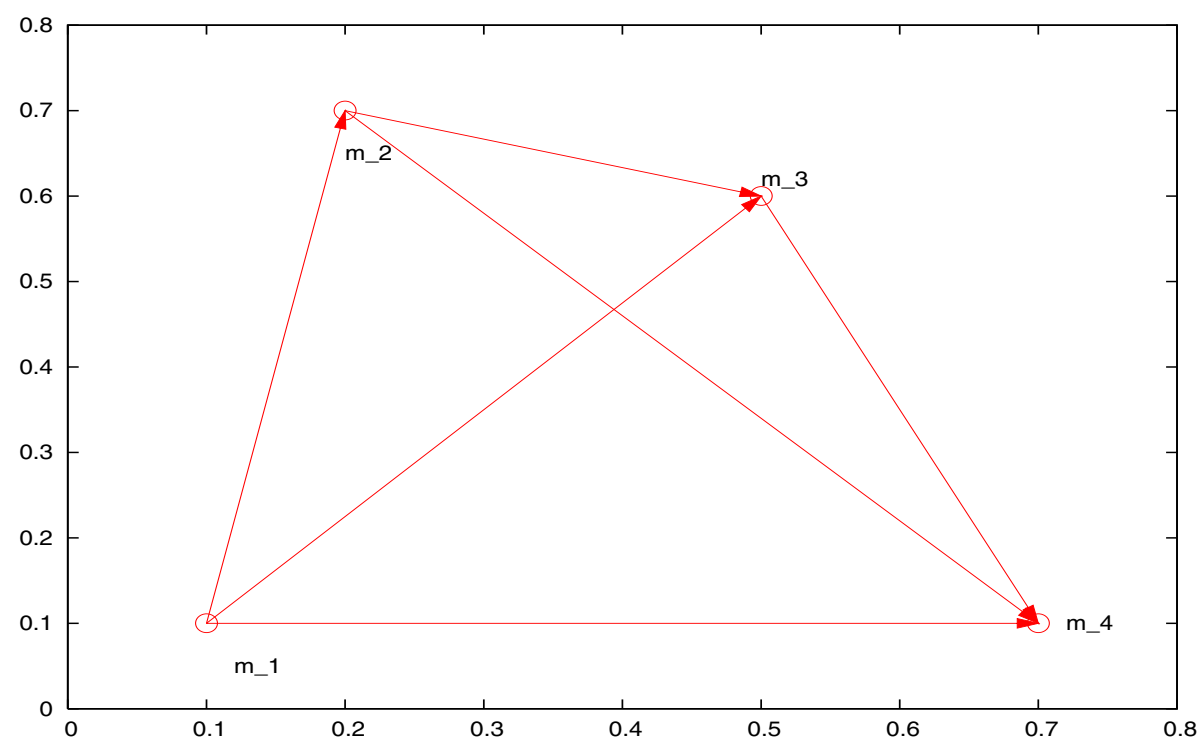

Figure 2. In the global regularization of Heggie, all the relative vectors are formally independent and regularised using KS.

After the introduction of the above transformation the old momenta are

$$
\mathbf{w}_{i}=\sum_{j=i+1}^{N} \mathbf{w}_{i j}-\sum_{j=1}^{i-1} \mathbf{w}_{j i} .
$$

Substituting these and applying the KS transformation to each conjugate pair $\left(\mathbf{w}_{i j}, \mathbf{r}_{i j}\right)$. one obtains the globally regular Hamiltonian

$$
\begin{aligned}
\Gamma & =g(\mathbf{r})\left[H\left(\mathbf{w}_{i j}, \mathbf{r}_{i j}\right)-E\right] \prod_{i=1}^{N-1} \\
& =g(\mathbf{Q})\left[H\left(\frac{\widehat{\mathbf{Q}}_{i j} \mathbf{P}_{i j}}{2 Q_{i j}^{2}}, \widehat{\mathbf{Q}}_{i j} \mathbf{Q}_{i j}\right)-E\right] .
\end{aligned}
$$

- Mikkola (1985) wrote a concise algorithm for $N$ body integration using Heggie's global method. This was possible by using

$$
g=1 / L
$$

instead of the originally (and customarily) used $g=\prod r_{i j}$.

- Mikkola \& Aarseth (1990), (1993) developed what is known as the chain method.

Suppose a chain of vectors connecting $N$ bodies has been selected. After re-labelling the bodies such that they are $1,2, \ldots, N$ along the chain, the generating function

$$
S=\sum_{i=1}^{N-1} \mathbf{W}_{k} \cdot\left(\mathbf{q}_{k+1}-\mathbf{q}_{k}\right)
$$

can be used to obtain the old momenta $\mathbf{p}_{k}=\partial S / \partial \mathbf{q}_{k}$ in terms of the new ones. In general, 


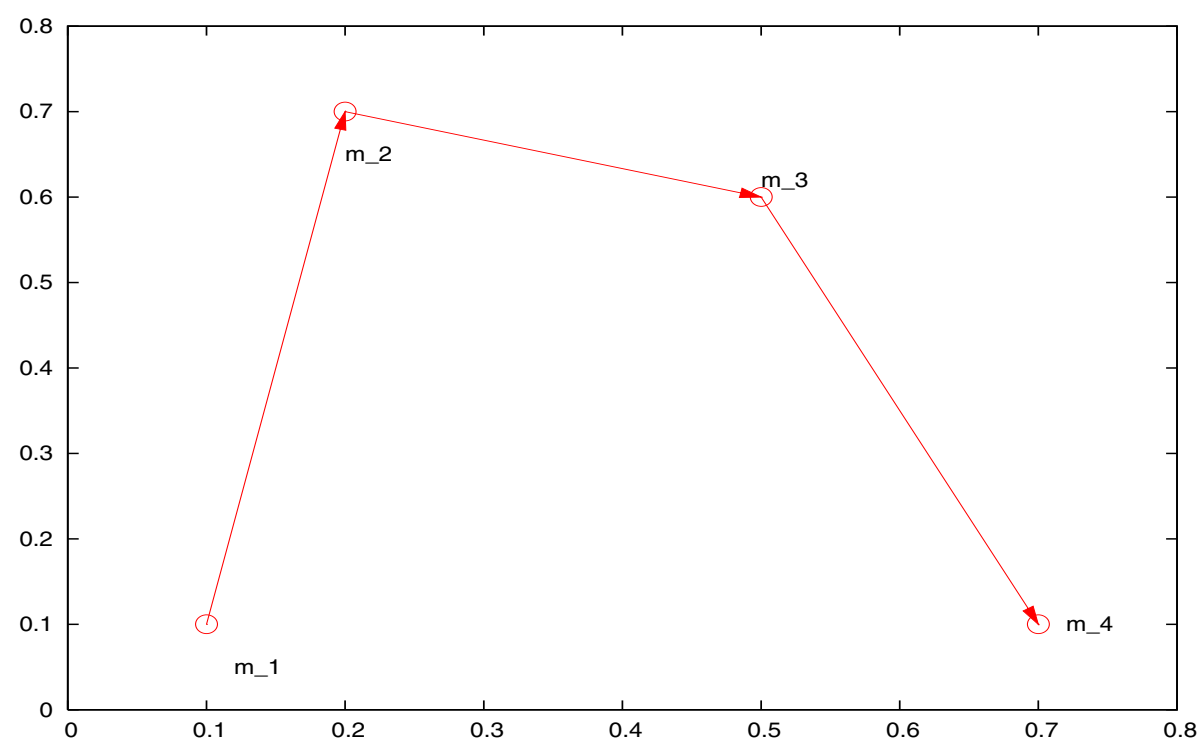

Figure 3. In the CHAIN method the chain vectors are regularised using KS. The chain also helps preventing serious roundoff errors.

$\mathbf{p}_{k}=\mathbf{W}_{k-1}-\mathbf{W}_{k} ; k=2, . ., N-1$, but the first and last take the form $\mathbf{p}_{1}=-\mathbf{W}_{1}$, $\mathbf{p}_{N}=\mathbf{W}_{N-1}$. By definition the corresponding chain vectors are given by $\mathbf{R}_{k}=\mathbf{q}_{k+1}-\mathbf{q}_{k}$. In the centre-of-mass system the Hamiltonian becomes

$$
H=\sum_{k=1}^{N-1} \frac{1}{2}\left(\frac{1}{m_{k}}+\frac{1}{m_{k+1}}\right) \mathbf{W}_{k}^{2}-\sum_{k=2}^{N} \frac{1}{m_{k}} \mathbf{W}_{k-1} \cdot \mathbf{W}_{k}-\sum_{k=1}^{N-1} \frac{m_{k} m_{k+1}}{R_{k}}-\sum_{1 \leqslant i \leqslant j-2} \frac{m_{i} m_{j}}{R_{i j}}
$$

where the non-chained distances are given by $R_{i j}=\left|\mathbf{r}_{j}-\mathbf{r}_{i}\right|=\left|\mathbf{q}_{j}-\mathbf{q}_{i}\right|=\left|\sum_{i \leqslant k^{\prime} \leqslant j-1} \mathbf{R}_{k^{\prime}}\right|$. Later different parts of this Hamiltonian, the kinetic energy, the chained and non-chained parts of the force function, respectively, will be denoted by $T, U_{c}$ and $U_{n c}$.

Substitution of the KS-transformations $\mathbf{R}_{k}=\widehat{\mathbf{Q}}_{k} \mathbf{Q}_{k}, \quad \mathbf{W}_{k}=\widehat{\mathbf{Q}}_{k} \mathbf{P}_{k} /\left(2 Q_{k}^{2}\right)$ gives the Hamiltonian in terms of the regularising variables $\mathbf{Q}_{k}, \mathbf{P}_{k}$. With the time transformation $d t=g d s$, with $g=1 /(T+U)$, one obtains the regularised Hamiltonian $\Gamma=g(H-E)=$ $(T-U-E) /(T+U)$ in the $(\mathbf{P}, \mathbf{Q}, s)$-system. The derivation of the equations of motion $\mathbf{P}_{k}{ }^{\prime}=-\partial \Gamma / \partial \mathbf{Q}_{k}, \quad \mathbf{Q}_{k}{ }^{\prime}=\partial \Gamma / \partial \mathbf{P}_{k}$, where primes denote differentiation with respect to $s$, is now possible remembering that $\partial \widetilde{U} / \partial \mathbf{Q}=2 \widehat{\mathbf{Q}}^{t} \mathbf{F}$, where $\mathbf{F}=\partial \widetilde{U} / \partial \mathbf{R}$.

\section{- Algorithmic Regularisation (AR).} (Mikkola \& Tanikawa 1999ab, Preto \& Tremaine 1999)

Case of two bodies: The Hamiltonian in extended phase space may be written

$$
H=T+b-U=\mathbf{p}^{2} / 2+b-m / r,
$$

where $\mathbf{p}$ is the momentum vector, $r$ the distance and the momentum of time $b=m / r-\mathbf{p}^{2} / 2=$ constant.

Instead of $H$ one may use a new Hamiltonian (easy to prove)

$$
\Lambda=\ln (T+b)-\ln (U),
$$




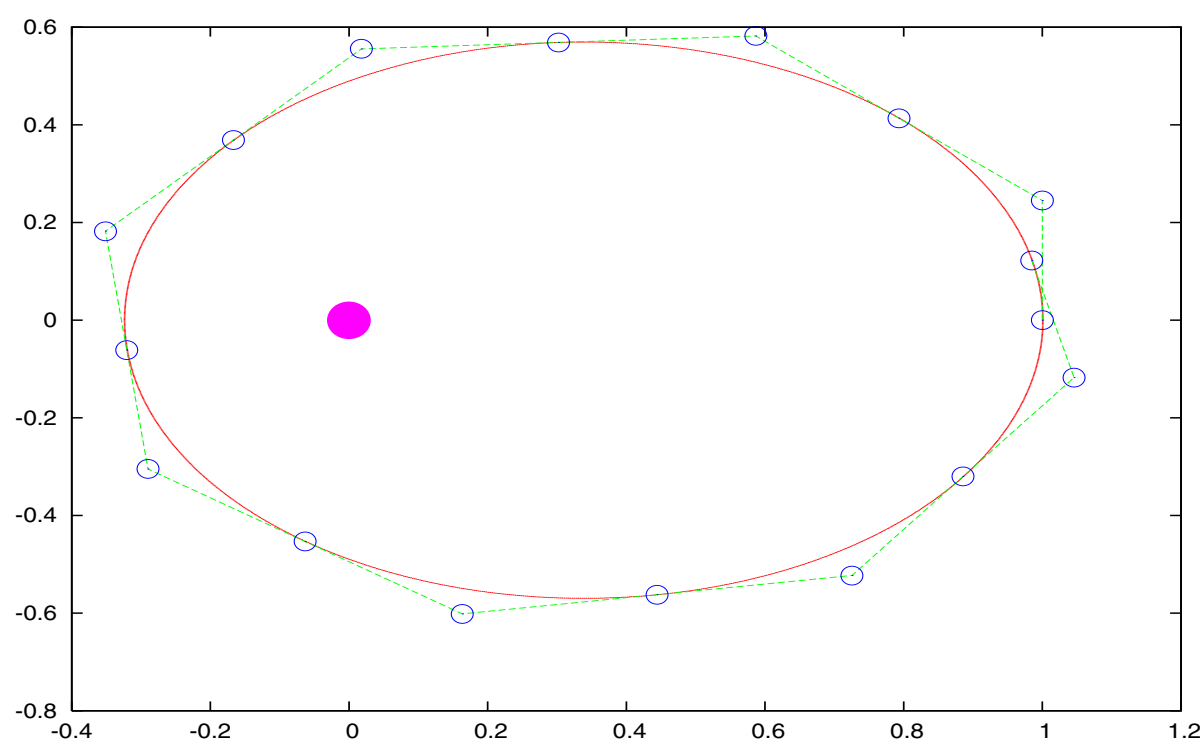

Figure 4. Logarithmic leapfrog trajectory for a two-body problem. The points outside the correct ellipse are the $\mathbf{r}_{\frac{1}{2}}$ points that are not to be considered physical coordinates, but only auxiliary quantities.

which gives equations of motion for coordinates $t$ and $\mathbf{r}$

$$
t^{\prime}=1 /\left(\mathbf{p}^{2} / 2+b\right) ; \quad \mathbf{r}^{\prime}=\mathbf{p} /\left(\mathbf{p}^{2} / 2+b\right)
$$

end for momenta $b$ and $\mathbf{p}$

$$
b^{\prime}=0 ; \quad \mathbf{p}^{\prime}=-\mathbf{r} / r^{2}
$$

One notices that a leapfrog algorithm is possible:

$$
\begin{aligned}
\delta t_{0} & =\frac{1}{2} h /\left(\mathbf{p}_{0}^{2} / 2+b\right) ; \quad t_{\frac{1}{2}}=t_{0}+\delta t_{0} \\
\mathbf{r}_{\frac{1}{2}} & =\mathbf{r}_{0}+\delta t_{0} \mathbf{p}_{0} \\
\mathbf{p}_{1} & =\mathbf{p}_{0}-h \mathbf{r}_{\frac{1}{2}} / r_{\frac{1}{2}}^{2} \\
\delta t_{1} & =\frac{1}{2} h /\left(\mathbf{p}_{1}^{2} / 2+b\right) ; \quad t_{1}=t_{\frac{1}{2}}+\delta t_{1} \\
\mathbf{r}_{1} & =\mathbf{r}_{\frac{1}{2}}+\delta t_{1} \mathbf{p}_{1} .
\end{aligned}
$$

A remarkable fact is that this simple, supposedly second order, algorithm gives a correct trajectory for the two-body motion. The only error is a phase error i.e. the time increment has a remainder of $O\left(h^{3}\right)$. The important fact is that even an exact collision orbit can be done by this algorithm. Consequently the logarithmic Hamiltonian leapfrog can be used for regularization without coordinate transformations.

- N-Body formulation(s)

\section{Logarithmic Hamiltonian:}

Let $T$ and $U$ be the kinetic and potential energies

$$
T=\sum_{k=1}^{N} \frac{m_{k}}{2} \mathbf{v}_{k}^{2} ; \quad U=\sum_{i<j \leqslant N} m_{i} m_{j} / r_{i j}+R(\mathbf{r}, t)
$$


such that the total energy is $E=T-U$ and use $B=-E$ for the binding energy.

The logarithmic Hamiltonian is

$$
\Lambda=\ln (T+B)-\ln (U)
$$

and the equations of motion

$$
\begin{aligned}
t^{\prime}=\partial \Lambda / \partial B ; & \mathbf{r}_{k}^{\prime}=\partial \Lambda / \partial \mathbf{p}_{k} \\
B^{\prime}=\partial \Lambda / \partial t ; & \mathbf{p}_{k}^{\prime}=\partial \Lambda / \partial \mathbf{r}_{k},
\end{aligned}
$$

allow the formation of the leapfrog algorithm. Define two subroutines: $\mathbf{X}(s)$ for moving coordinates over a step $=s$

$$
\mathbf{X}(s): \quad \delta t=s /(T+B) ; \quad \mathbf{r}_{k} \rightarrow \mathbf{r}_{k}+\delta t \mathbf{v}_{k}, \quad t \rightarrow t+\delta t
$$

and routine $\mathbf{V}(s)$ for velocity jumps

$$
\mathbf{V}(s): \quad \delta \tau=s / U ; \quad \mathbf{v}_{k} \rightarrow \mathbf{v}_{k}+\delta \tau \partial U / \partial \mathbf{r}_{k} ; \quad B \rightarrow B+\delta \tau \partial U / \partial t
$$

The leapfrog algorithm for $n$ steps can then be symbolised as

$$
\mathbf{X}(h / 2)(\mathbf{V}(h) \mathbf{X}(h))^{n-1} \mathbf{V}(h) \mathbf{X}(h / 2) .
$$

This leads in principle to an algorithmically regularised method, results of which could be improved to high precision using the extrapolation method (Bulirsch \& Stoer 1966.) Here the main problem that remains is roundoff. The cure for this is the use of the chain.

\section{- Time Transformed Leapfrog (TTL):}

Recently Mikkola \& Aarseth (2002) suggested an alternative to the logH method. Here one introduces a new variable $\omega$ and a function $\Omega(\mathbf{r})$ such that $\omega(0)=\Omega(0)$ and the values of $\omega$ are obtained via the differential equation $\dot{\omega}=\dot{\Omega}(\mathbf{r})=\partial \Omega / \partial \mathbf{r} \cdot \mathbf{v}$. This allows one to write the coordinate equations in the form

$$
\begin{aligned}
t^{\prime} & =1 / \omega \\
\mathbf{r}^{\prime} & =\mathbf{v} / \omega
\end{aligned}
$$

and the velocity equations

$$
\begin{aligned}
\mathbf{v}^{\prime} & =\frac{\partial U}{\partial \mathbf{r}} / \Omega(\mathbf{r}) \\
\omega^{\prime} & =\frac{\partial \Omega}{\partial \mathbf{r}} \cdot \mathbf{v} / \Omega .
\end{aligned}
$$

This formulation also allows a leapfrog construction and if $\Omega \sim 1 / r_{\min }$ when $r_{\min } \rightarrow 0$, then the algorithm is asymptotically the same as the $\log H$ leapfrog. This fact makes it possible to regularise the close approaches of small bodies which do not affect considerably the value of the potential.

\section{- Algorithmic Regularization Chain}

One forms a chain of particles such that the shortest relative vectors are in the chain (Mikkola \& Aarseth 1993). It is necessary to stress that the main purpose of using the chain structure in this method is to reduce the effect of roundoff error. In this the chain is effective.

Let us collect the chain coordinates

$$
\mathbf{X}_{k}=\mathbf{r}_{i_{k}}-\mathbf{r}_{j_{k}}
$$


in the vector

$$
\mathbf{X}=\left(\mathbf{X}_{1}, \mathbf{X}_{2}, \ldots, \mathbf{X}_{N-1}\right)
$$

and let the corresponding velocities be

$$
\mathbf{V}=\left(\mathbf{V}_{1}, \mathbf{V}_{2}, \ldots, \mathbf{V}_{N-1}\right) .
$$

Then the Newtonian equations of motion may be formally written

$$
\begin{aligned}
\dot{\mathbf{X}} & =\mathbf{V} \\
\dot{\mathbf{V}} & =\mathbf{A}(\mathbf{X})+\mathbf{f},
\end{aligned}
$$

where $\mathbf{A}$ is the N-body acceleration and $\mathbf{f}$ is some external acceleration (e.g.due to other bodies).

One may use the two equivalent time transformations (Mikkola \& Merritt 2006).

$$
d s=[\alpha(T+B)+\beta \omega+\gamma] d t=[\alpha U+\beta \Omega+\gamma] d t,
$$

where $s$ is a new independent variable, $B$ is the binding energy $B=-E, \alpha, \beta$ and $\gamma$ are adjustable constants, $\Omega$ is an optional function of the coordinates $\Omega=\Omega(\mathbf{X})$. The initial value $\omega(0)=\Omega(0)$ and the differential equation

$$
\dot{\omega}=\frac{\partial \Omega}{\partial \mathbf{X}} \cdot \mathbf{V},
$$

determines the value of $\omega$ (actually $\omega(t)=\Omega(t)$ along the exact solution).

It is possible to divide the equations of motion into two categories (when derivatives with respect to the new independent variable $s$ are denoted by a prime).

Coordinate equations:

$$
\begin{aligned}
t^{\prime} & =1 /(\alpha(T+B)+\beta \omega+\gamma) \\
\mathbf{X}^{\prime} & =t^{\prime} \mathbf{V}
\end{aligned}
$$

Velocity equations:

$$
\begin{aligned}
\tilde{t}^{\prime} & =1 /(\alpha U+\beta \Omega+\gamma) \\
\mathbf{V}^{\prime} & =\tilde{t}^{\prime}(\mathbf{A}+\mathbf{f}) \\
\omega^{\prime} & =\tilde{t}^{\prime} \frac{\partial \Omega}{\partial \mathbf{X}} \cdot \mathbf{V} \\
B^{\prime} & =-\tilde{t}^{\prime} \frac{\partial T}{\partial \mathbf{V}} \cdot f
\end{aligned}
$$

In these equations the right hand sides do not depend on the variables at the left hand side. Consequently it is possible to construct a regular leapfrog algorithm for obtaining the solutions (Mikkola \& Tanikawa 1999ab, Mikkola \& Aarseth 2003, Preto \& Tremaine 1999).

The time transformation thus introduced regularises the two-body collisions if one uses the simple leapfrog algorithm as a basic integrator (results of which can, and must, be improved using an extrapolation method (e.g. Bulirsch \& Stoer 1966). [This is why the method is called Algorithmic Regularization.]

For the case of velocity dependent perturbation $\mathbf{f}=\mathbf{f}(\mathbf{X}, \mathbf{V})$, which occurs e.g if one introduces relativistic Post-Newtonian terms, related algorithms were discussed by (Mikkola \& Merritt 2006).

In the presence of external perturbations the binding energy evolves according to

$$
\dot{B}=-\frac{\partial T}{\partial \mathbf{V}} \cdot \mathbf{f}
$$


The leapfrog for the chain vectors $\mathbf{X}$ and $\mathbf{V}$ can be written as the two mappings

$$
\begin{aligned}
\mathbf{X}(s): & \\
\delta t & =s /(\alpha(T+B)+\beta \omega+\gamma) \\
t & =t+\delta t \\
\mathbf{X} & \rightarrow \mathbf{X}+\delta t \mathbf{V} \\
\mathbf{V}(s): & \\
\widetilde{\delta t} & =s /(\alpha U+\beta \Omega+\gamma) \\
\mathbf{V} & \rightarrow \mathbf{V}+\widetilde{\delta t}(\mathbf{A}+\mathbf{f}) \\
B & \rightarrow B+\widetilde{\delta t}\left\langle\frac{\partial T}{\partial \mathbf{V}}\right\rangle \cdot \mathbf{f} \\
\omega & \rightarrow \omega+\widetilde{\delta t} \frac{\partial \Omega}{\partial \mathbf{X}} \cdot\langle\mathbf{V}\rangle,
\end{aligned}
$$

where $\left\langle\frac{\partial T}{\partial \mathbf{V}}\right\rangle$ and $\langle\mathbf{V}\rangle$ are the averages over the advancement of $\mathbf{V}$.

The leapfrog with the above maps reads

$$
\mathbf{X}(h / 2)(\mathbf{V}(h) \mathbf{X}(h))^{n-1} \mathbf{V}(h) \mathbf{X}(h / 2),
$$

for a macro-step of length $=n h$.

\section{- Alternatives for the time-transformation}

If one takes

$$
\Omega=\sum_{i<j} \Omega_{i j} / r_{i j} \text { and } \Omega_{j}=m_{i} m_{j},
$$

then $\alpha=0, \beta=1, \gamma=0$ is mathematically equivalent to $\alpha=1, \beta=\gamma=0$ as was shown in Mikkola \& Aarseth (2002). However, numerically these are not equivalent, but the $\log \mathrm{H}$ alternative is much more stable. On the other hand, as noted above, it is desirable to get step size shortening (and thus regularization) also for encounters of small bodies and thus some function $\Omega$ should be used.

The increase the numerical stability for strong interactions of big bodies and also smooth the encounters of small bodies one may use $\alpha=1, \beta \neq 0$ and

$$
\Omega_{i j}=\left\{\begin{array}{l}
=C_{m} ; \text { if } m_{i} * m_{j}<\epsilon C_{m} \\
=0 ; \text { otherwise }
\end{array}\right.
$$

where $C_{m}=\sum_{i<j} m_{i} m_{j} /(N(N-1) / 2)$ is the mean mass product and $\epsilon$ an adjustable parameter (usually $\epsilon \sim 10^{-3}$ or smaller, may be recommended).

\section{Numerical Experiments}

Here we discuss only results from a 7-body system with masses $m_{1}=1, m_{2}=.1$ and 5 masses of $m_{k}=.001 k=3, \ldots, 7$. Experiments were made with a KS-CHAIN and an ARCHAIN code. Integrations were carried out with and without relativistic perturbations and output was done with and without requiring exact output time (slower, because of iteration to exact time). It was also checked that the solutions obtained were similar. In all cases the accuracies were comparable $\left(|\delta E / L|<10^{-12}\right)$, but invariably the ARCHAIN was faster by a factor of few. This does not necessarily mean that this is always the case, but seems to be typical for large mass ratios. 


\section{Discussion and Conclusion}

The various alternatives to write regular N-Body algorithms (the KS and AR) were briefly reviewed. Numerical experiments have shown that the new AR method is often efficient even when the mass ratios are large (in this case the KS based algorithms are more difficult to use).

There is still a problem, related to the TTL (and AR-CHAIN) algorithm: the auxiliary quantity $\omega$ is obtained by summation of increments $\omega_{\text {new }}=\omega_{\text {old }}+\delta \omega$, where the increments $\delta \omega$ can be large and of varying sign. There is often considerable cancellation and loss of significant figures. This is one of the most important remaining problems in the AR-algorithm and needs to be investigated in future research.

\section{References}

Aarseth, S. J. 1972, in Gravitational N-body Problem, proceedings of IAU colloquium No. 10, ed. M. Lecar, Reidel, Dordrecht, pp.373-387.

Aarseth, S. J. 2003, Gravitational N-Body Simulations, Cambridge Univ. Press, Cambridge Aarseth, S. J. \& Zare, K. 1974, Celestial Mechanics, 10, 185

Bulirsch, R. \& Stoer, J. 1966, Numerical Mathematics, 8, 1

Heggie, D. C. 1974, Celestial Mechanics, 10, 217

Kustaanheimo, P. \& Stiefel, E. 1965, J. Reine Angew. Math., 218, 204

Levi-Civita, T. 1920, Acta Mathematica, 42, 99

Mikkola, S. 1985, MNRAS, 215, 171

Mikkola, S. \& Aarseth, S. J. 1990, Celestial Mechanics and Dynamical Astronomy, 47, 375

Mikkola, S. \& Aarseth, S. J. 1993, Celestial Mechanics and Dynamical Astronomy, 57, 439

Mikkola, S. \& Aarseth, S. 2002, Celestial Mechanics and Dynamical Astronomy, 84, 343

Mikkola, S. \& Merritt, D. 2006, MNRAS, 372, 219

Mikkola, S. \& Tanikawa, K. 1999a, Celestial Mechanics and Dynamical Astronomy, 74, 287

Mikkola, S. \& Tanikawa, K. 1999b, MNRAS, 310, 745

Preto, M. \& Tremaine, S. 1999, AJ, 118, 2532

Siegel. C. L. 1956, Vorlesungen über Himmelsmechanik, Springer, Berlin-Göttingen-Heidelberg. Stiefel, E. L. \& Scheifele, G. 1971, Linear and Regular Celestial Mechanics, Springer, Berlin.

Szebehely, V. \& Peters, C. F. 1967, AJ, 72, 876.

von Hoerner, S. 1960, Z. Astrophys., 50, 184

von Hoerner, S. 1963, Z. Astrophys., 57, 47

Zare, K. 1974, Celestial Mechanics, 10, 207 